Article

\title{
The Effect of Calcium and Halide Ions on the Gramicidin A Molecular State and Antimicrobial Activity
}

\author{
Kathleen D. Carillo ${ }^{1,2,3, \dagger}$, Chi-Jen Lo ${ }^{4,+}{ }^{\circledR}$, Der-Lii M. Tzou ${ }^{3,5,+} \oplus$, Yi-Hung Lin ${ }^{6}$, \\ Shang-Ting Fang ${ }^{7}$, Shu-Hsiang Huang ${ }^{7}$ and Yi-Cheng Chen ${ }^{7, *} \mathbb{C}$ \\ 1 Taiwan International Graduate Program, Sustainable Chemical Science and Technology (SCST), \\ Academia Sinica, Nankang, Taipei 11529, Taiwan; kjcarillo@yahoo.com \\ 2 The Department of Applied Chemistry, National Chiao-Tung University, Hsinchu 30013, Taiwan \\ 3 Institute of Chemistry, Academia Sinica, Nankang, Taipei 11529, Taiwan; tzougate@gate.sinica.edu.tw \\ 4 Metabolomics Core Laboratory, Healthy Aging Research Center, Chang Gung University, \\ Taoyuan 333, Taiwan; chijenlo@gmail.com \\ 5 Department of Applied Chemistry, National Chia-Yi University, Chia-Yi 60004, Taiwan \\ 6 Experimental Facility Division, Industrial Application Group, National Synchrotron Radiation Research \\ Center, Hsinchu Science Park, Hsinchu 30076, Taiwan; yihung@nsrrc.org.tw \\ 7 Department of Medicine, MacKay Medical College, New Taipei City 252, Taiwan; \\ farmting@hotmail.com (S.-T.F.); sshuang33@mmc.edu.tw (S.-H.H.) \\ * Correspondence: chen15@mmc.edu.tw \\ + These authors contributed equally to this work.
}

Received: 15 July 2020; Accepted: 24 August 2020; Published: 27 August 2020

\begin{abstract}
Gramicidin A (gA) forms several convertible conformations in different environments. In this study, we investigated the effect of calcium halides on the molecular state and antimicrobial activity of gramicidin A. The molecular state of gramicidin A is highly affected by the concentration of calcium salt and the type of halide anion. Gramicidin A can exist in two states that can be characterized by circular dichroism (CD), mass, nuclear magnetic resonance (NMR) and fluorescence spectroscopy. In State 1, the main molecular state of gramicidin A is as a dimer, and the addition of calcium salt can convert a mixture of four species into a single species, which is possibly a left-handed parallel double helix. In State 2, the addition of calcium halides drives gramicidin A dissociation and denaturation from a structured dimer into a rapid equilibrium of structured/unstructured monomer. We found that the abilities of dissociation and denaturation were highly dependent on the type of halide anion. The dissociation ability of calcium halides may play a vital role in the antimicrobial activity, as the structured monomeric form had the highest antimicrobial activity. Herein, our study demonstrated that the molecular state was correlated with the antimicrobial activity.
\end{abstract}

Keywords: gramicidin A; halide; molecular state; antimicrobial activity; dissociation

\section{Introduction}

Gramicidin is an antimicrobial peptide that is a byproduct of Bacillus brevis during sporulation [1]. Gramicidin contains alternating L- and D-amino acids in its primary sequence and has three isoforms - gramicidin A $(\mathrm{gA})$, gramicidin $\mathrm{B}(\mathrm{gB})$ and gramicidin $\mathrm{C}(\mathrm{gC})$-in which residue 11 is Trp for gA, Phe for $\mathrm{gB}$ and Tyr for gC [2,3]. Gramicidin adopts a number of different conformations in different environmental conditions [4-7]. In organic solvents such as methanol, gramicidin forms a $\beta$-sheet-like double-strand helical structure, in which two monomers are interwound similarly as DNA double helices $[4,6,8]$. This double-strand helical dimer has four distinct conformations that can be 
either parallel or antiparallel, left- or right-handed double-stranded helices [6-9]. These double-helical conformers are interconvertible and have distinct circular dichroism (CD) spectra $[6,8]$. On the other hand, in polar solvents such as trifluoroethanol (TFE), gramicidin forms a single-stranded and right-handed helical monomer [4,10-12]. Two of the helical monomers can form a head-to-head channel dimer in a lipid environment $[4,11,12]$. This channel can specifically translocate small monovalent cations such as $\mathrm{H}^{+}, \mathrm{Na}^{+}$and $\mathrm{K}^{+}$across membranes [4,12-14].

Gramicidin's double-stranded helical dimer can interact with monovalent or divalent cations [15-21]. The complex of gramicidin A/monovalent cation exists in a left-handed antiparallel double-helical conformation in methanol $[15,16]$ and has a distinct CD spectrum compared to that of the ion-free form [17]. The binding mechanism is a cooperative mode, and the binding affinities are roughly related to the size of the monovalent cations. The order of the binding affinities is $\mathrm{Cs}>\mathrm{Rb}>>\mathrm{K}>\mathrm{Li}$ [17]. Previous studies demonstrated that divalent cations could cause the gramicidin A mixture in methanol to form one specific conformation [18,19]. The CD spectrum of the gramicidin A/divalent cation complex is very different from that of the gramicidin $\mathrm{A} /$ monovalent cation complex in methanol [19]. The structure of the gramicidin A/Ca complex determined by the solution NMR technique appears to be a left-handed parallel double helical conformation with Ca bound at the $\mathrm{N}$-terminal mouth of the double helical dimer [20].

Conversion between conformers has been studied in lipid environments [21-25]. In membrane lipid environments, the rate of conversion from the double-stranded helical dimer into the channel dimer is dependent on environmental factors, such as temperature and ionic strength [21-23]. Both CD and fluorescence spectroscopies have been used to examine the rates of conversion of the double-stranded helical dimer to the single-stranded helical dimer in synthetic lipid membranes [21,23]. However, the conversion between the double-strand helical conformers in organic solvents and different ions has not been studied.

Gramicidin A is a short peptide antibiotic effective against bacteria and fungi [26]. The antimicrobial activity of gramicidin A has been associated with the disruption of membrane lipids [27]. We have previously demonstrated that the antimicrobial activity of gramicidin A may also be associated with the formation of free radicals via the disruption of $\mathrm{NADH} / \mathrm{NAD}^{+}$synthesis [28]. Recently, the antimicrobial function of gramicidin A was also linked with the formation of ion channels [29]. However, the relationship between antimicrobial activity and molecular state is not entirely clear.

In the present study, we revisited and characterized the conformational and molecular states of gA in the presence of different calcium and halide ions using circular dichroism (CD), nuclear magnetic resonance (NMR) and state-of-art mass spectroscopy, and found that gramicidin A exists in two molecular states in the presence of calcium halides. In State 1, the main effect of calcium halides on gramicidin A is to convert the conformers into one single conformation. In State 2, calcium halides can induce a dissociation of the gramicidin A dimer into monomers and the denaturation of the gramicidin A monomer. A new finding revealed that the different halide anions may play a role in the molecular state and conformation of gramicidin A. The molecular state and conformation induced by calcium halide can further influence the antimicrobial activity of gramicidin A.

\section{Results}

\subsection{Circular Dichroism Spectroscopy}

Gramicidin A adopts several conformers in methanol [4,6-8]. We characterized the conformational and molecular states of gramicidin $\mathrm{A}$ in the presence of calcium halides and in methanol using several spectroscopic techniques including $C D, N M R$ and mass spectroscopy.

Figure $1 \mathrm{~A}, \mathrm{~B}$ shows the $\mathrm{CD}$ spectra for $100 \mu \mathrm{M}$ gramicidin A titrated with $\mathrm{CaCl}_{2}$ and $\mathrm{CaBr}_{2}$. For $\mathrm{CaBr}_{2}$, the $\mathrm{CD}$ spectra below $220 \mathrm{~nm}$ became noisy at the concentration $\geq 0.2 \mathrm{mM}$ due to the strong adsorption from the $\mathrm{Br}^{-}$anion. Therefore, we were unable to collect $\mathrm{CD}$ spectra from 200 to $220 \mathrm{~nm}$. A similar result was also obtained when we titrated gramicidin A with $\mathrm{CaI}_{2}$. An even stronger 
absorption arising from the $\mathrm{I}^{-}$anion prevented us from collecting any reasonable CD spectra. Hence, we were unable to study the effect of $\mathrm{CaI}_{2}$ on the gramicidin $\mathrm{A}$ conformation using $\mathrm{CD}$ spectroscopy.
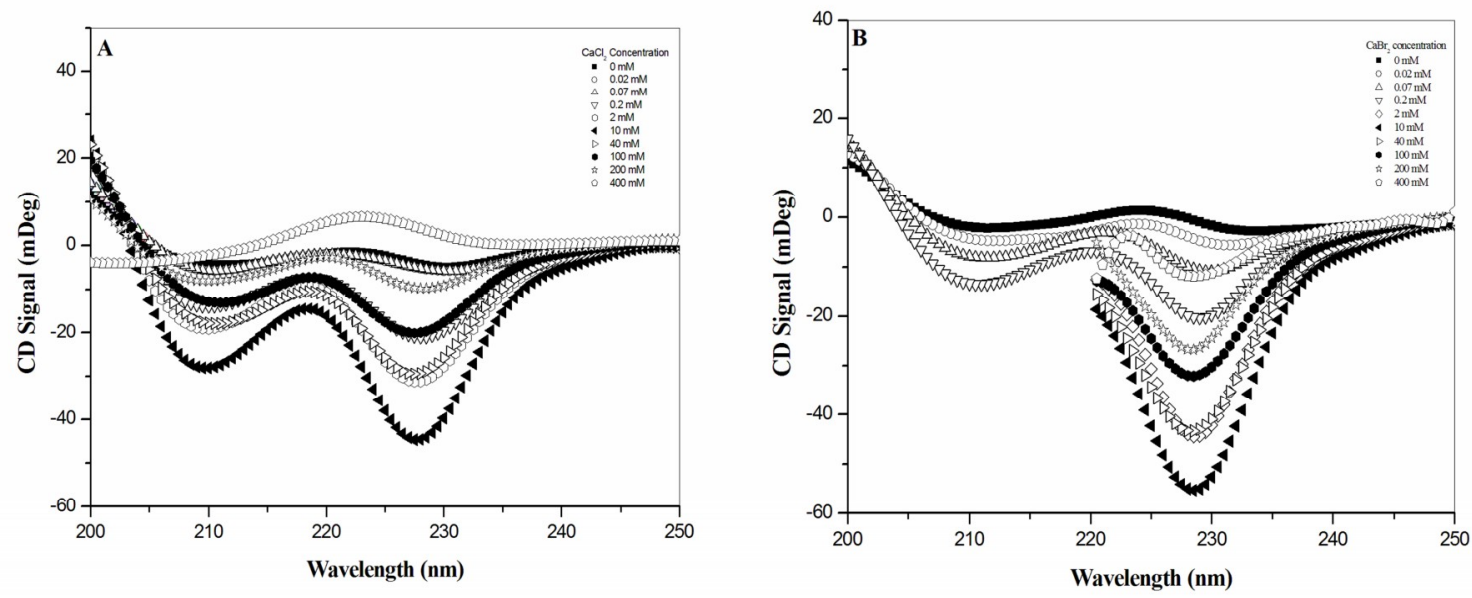

Figure 1. Circular dichroism (CD) spectra of gramicidin $\mathrm{A}$ in the presence of $\mathrm{CaCl}_{2}$ and $\mathrm{CaBr}_{2}$. One hundred micromolar gramicidin A was titrated with either $(\mathbf{A}) \mathrm{CaCl}_{2}$ or $(\mathbf{B}) \mathrm{CaBr}_{2}$ in methanol. All CD spectra were recorded from 200 to $250 \mathrm{~nm}$.

At salt concentrations $\leq 10 \mathrm{mM}$, the $\mathrm{CD}$ spectra showed similar spectral patterns, with the two negative peaks located at 208 and $228 \mathrm{~nm}$ for both $\mathrm{CaCl}_{2}$ and $\mathrm{CaBr}_{2}$. The intensity of the two negative peaks increases with an increase in the salt concentration and reaches its maximum at around $10 \mathrm{mM}$. At salt concentrations $>10 \mathrm{mM}$, these two negative peaks gradually converted into a single broadened positive peak located at $\sim 225 \mathrm{~nm}$. The change in intensity was different between the $\mathrm{Cl}^{-}$and $\mathrm{Br}^{-}$ anions. For $\mathrm{CaCl}_{2}$, the two negative peaks converted into a positive peak at a salt concentration higher than $300 \mathrm{mM}$, whereas the two negative peaks did not convert into a positive peak at $400 \mathrm{mM}$ for $\mathrm{CaBr}_{2}$. Figure 2 shows the titration curve of the intensity at $228 \mathrm{~nm}$ vs. salt concentration. According to the titration curves (Figure 2), gramicidin A may exist in two states for both $\mathrm{CaCl}_{2}$ and $\mathrm{CaBr}_{2}$. The CD results suggest that gramicidin A may adopt different conformations in these two states for $\mathrm{CaCl}_{2}$ and $\mathrm{CaBr}_{2}$.

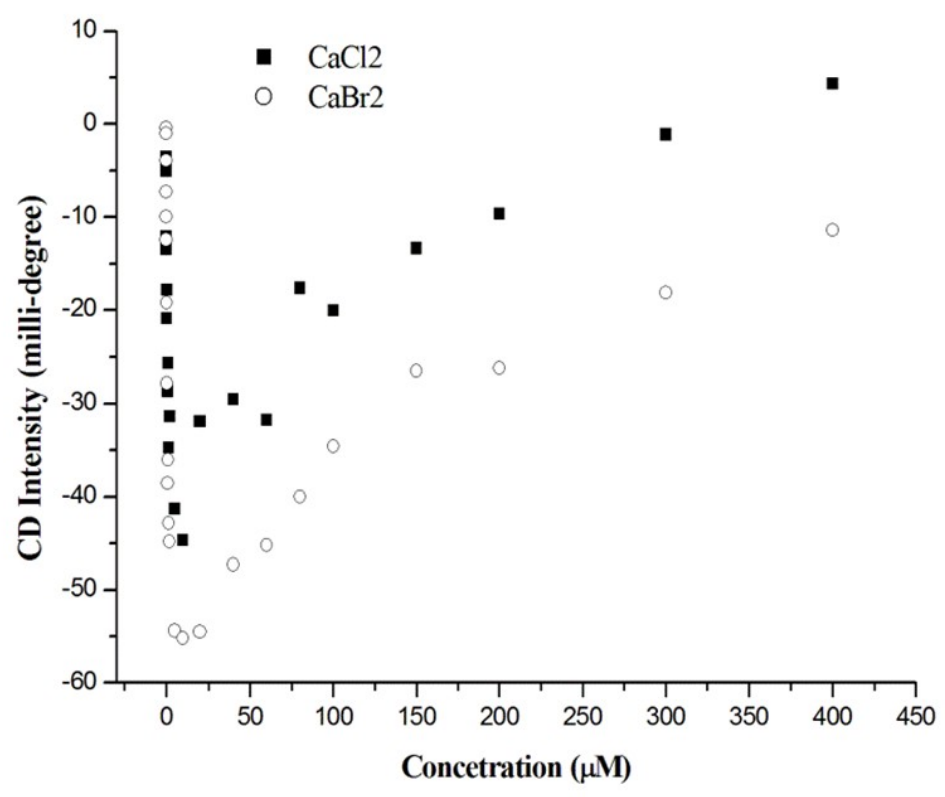

Figure 2. The intensity of the CD signal at $228 \mathrm{~nm}$ vs. the Ca salt concentration. The titration curves for one hundred micromolar gramicidin A titrated with $\mathrm{CaCl}_{2}(\mathbf{\square})$ and $\mathrm{CaBr}_{2}(\bigcirc)$ in methanol, respectively. 


\subsection{Nuclear Magnetic Resonance Spectroscopy}

The conformation of gramicidin A in the presence of calcium halide salts was examined using 1D proton NMR spectroscopy. The 1D proton NMR spectra of gramicidin A in the presence of $\mathrm{CaCl}_{2}, \mathrm{CaBr}_{2}$ and $\mathrm{CaI}_{2}$ are shown in Figures 3 and 4, and Figure $\mathrm{S} 1$ in Supplementary Information, respectively. Two spectral features in the NMR spectrum are particularly interesting. The chemical shifts at 8.0-8.2 ppm were assigned as the $\mathrm{N}$-terminal formyl group $[19,30]$. The resonance assignment for the chemical shifts at 10.0-10.5 ppm was not determined in this study and required further 2D experiments.

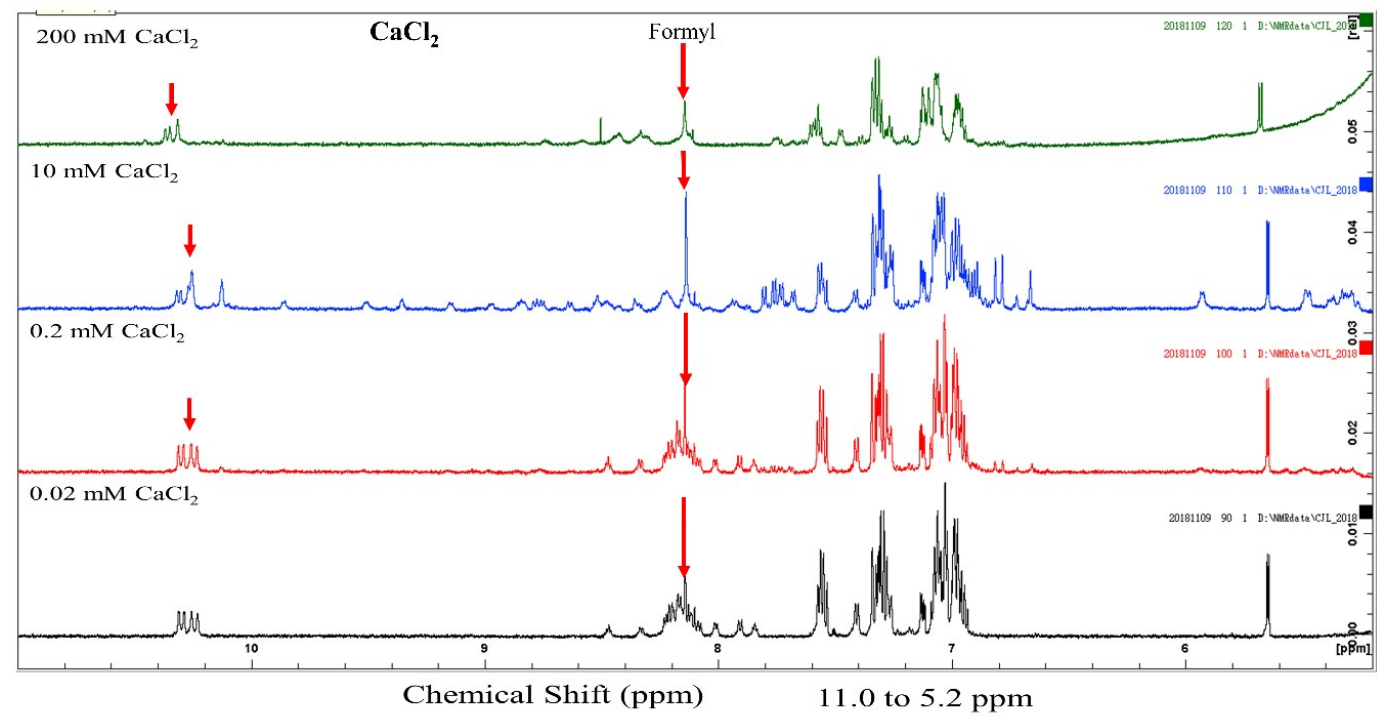

Figure 3. Nuclear magnetic resonance (NMR) spectra of gramicidin $\mathrm{A}$ in the presence of $\mathrm{CaCl}_{2}$. From bottom to top, the NH region, 5.0-10.5 ppm, is shown for $100 \mu \mathrm{M}$ gramicidin A with $0.02,0.2$, 10 and $200 \mathrm{mM} \mathrm{CaCl}_{2}$ in $90 \%$ d4-methanol/10\% methanol.

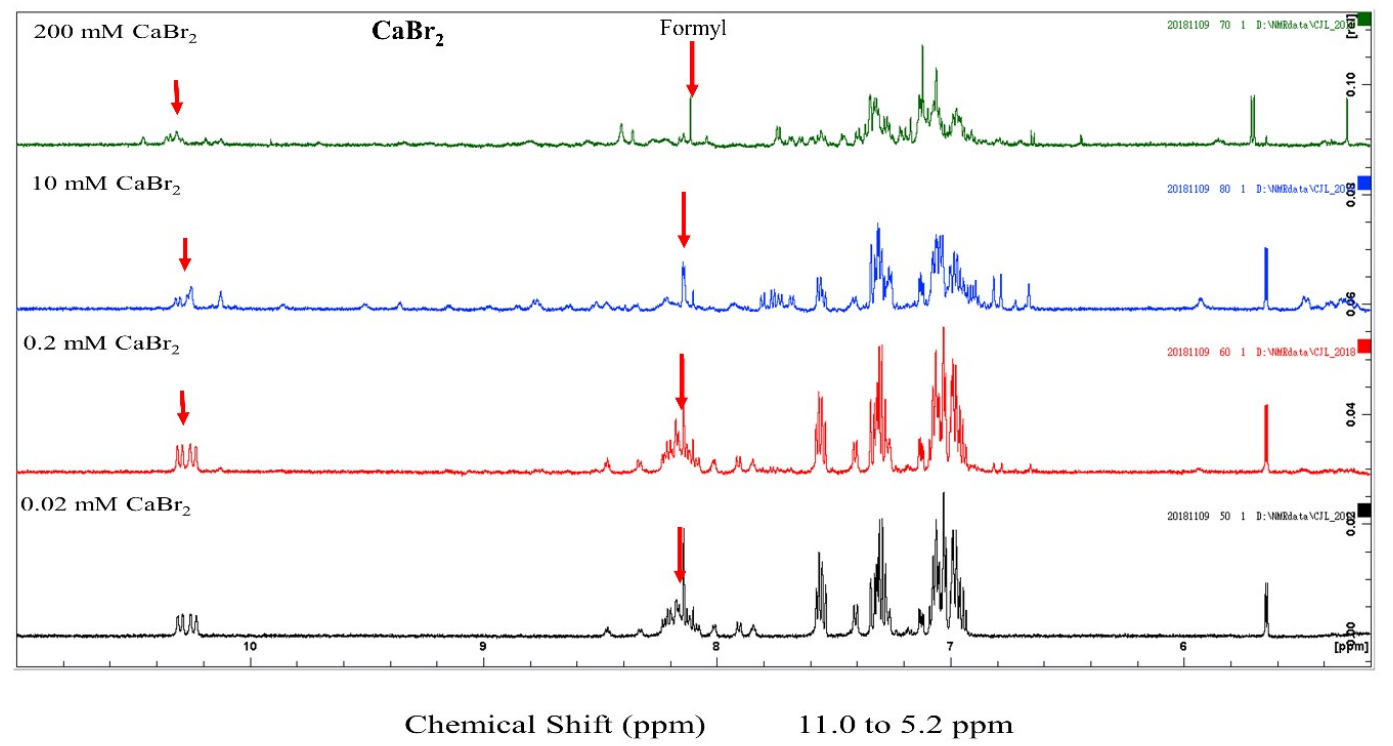

Figure 4. NMR spectra of gramicidin $\mathrm{A}$ in the presence of $\mathrm{CaBr}_{2}$. From bottom to top, the $\mathrm{NH}$ region, 5.0-10.5 ppm, is shown for $100 \mu \mathrm{M}$ gramicidin A with $0.02,0.2,10$ and $200 \mathrm{mM} \mathrm{CaBr}_{2}$ in $90 \%$ d4-methanol/10\% methanol.

At a low salt concentration, i.e., $0.2 \mathrm{mM}$, the proton signal of the formyl group was multiple resonance for $\mathrm{CaCl}_{2}, \mathrm{CaBr}_{2}$ and $\mathrm{CaI}_{2}$, indicating that a mixture of conformers is present under this 
condition. Unlike the resonance pattern of the formyl group at a concentration of $0.2 \mathrm{mM}$, the resonance of the formyl proton became a single peak at a concentration of $10 \mathrm{mM}$ for $\mathrm{CaCl}_{2}, \mathrm{CaBr}_{2}$ and $\mathrm{CaI}_{2}$, indicating that a single conformation was present. The dispersion of these NMR spectra was well resolved at 5.0 10.0 ppm compared that of to the spectra obtained at a concentration of $0.2 \mathrm{mM}$. The well-resolved peaks at 10-10.5 ppm were not well separated compared to those at $0.2 \mathrm{mM}$. According to a previous study [20], gramicidin A may form a left-handed parallel double helix at $10 \mathrm{mM}$ calcium salt.

At a high salt concentration, i.e., $200 \mathrm{mM}$, the NMR spectra of gramicidin A were very different compared to those obtained at $10 \mathrm{mM}$ or $0.2 \mathrm{mM}$ and dependent on the different types of halide anion as well. At a concentration of $200 \mathrm{mM} \mathrm{CaCl}_{2}$, the individual peaks in the NMR spectrum were less dispersed compared to those in the NMR spectrum of gramicidin A at $10 \mathrm{mM} \mathrm{CaCl}_{2}$. A previous study has hypothesized that gramicidin A may possibly exist in a mixture of unstructured and structured monomers under this condition [19]. The NMR spectrum for $\mathrm{CaI}_{2}$ was even more separated, and peaks for a specific type of amino acid even coincided, indicating that gramicidin A may be further denatured rather than forming a structured/unstructured monomer in $\mathrm{CaBr}_{2}$ and $\mathrm{CaI}_{2}$. Clearly, the types of halide anions have different impacts on the conformation of gramicidin A at high salt concentrations.

\subsection{Mass Spectroscopy}

To further characterize the molecular state of gramicidin A at the different concentrations of calcium salt and with the different types of halide anion, we applied MALDI-TOF mass spectrometry to study the molecular state of gramicidin A. Figures 5 and 6 show the mass spectra of gramicidin $\mathrm{A}$ with $\mathrm{CaCl}_{2}$ and $\mathrm{CaBr}_{2}$ at various concentrations, respectively. The mass spectra of gramicidin $\mathrm{A}$ treated with $\mathrm{CaI}_{2}$ are delineated in Figure S2.
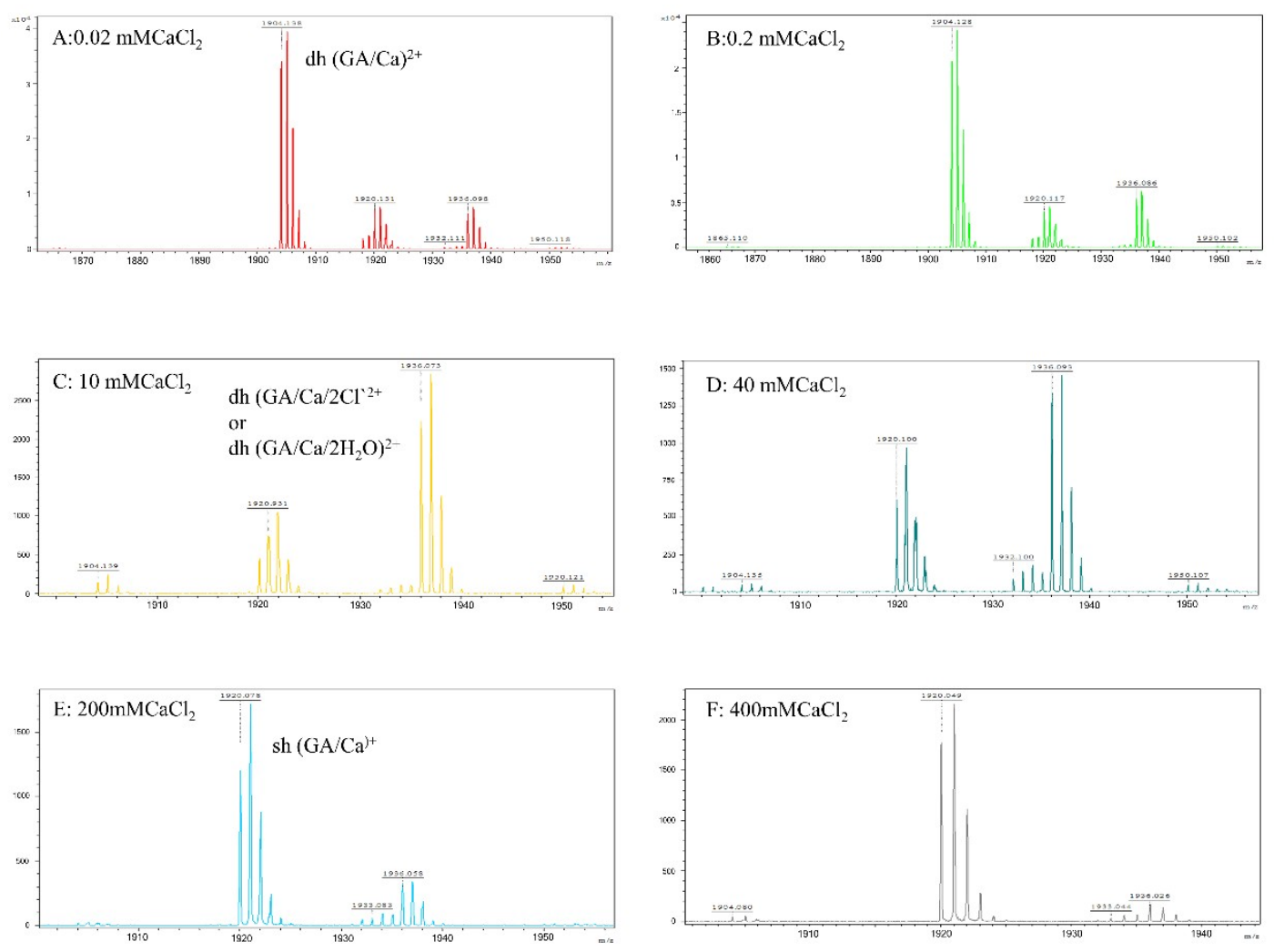

Figure 5. Mass spectra of gramicidin $\mathrm{A}$ in the presence of $\mathrm{CaCl}_{2}$. The samples containing $100 \mu \mathrm{M}$ gramicidin A with $\mathrm{CaCl}_{2}$ at 0.02 (A), 0.2 (B), 10 (C), 40 (D), 100 (E) and $400 \mathrm{mM}(\mathbf{F})$ in methanol were mixed with the matrix for mass analyses. 

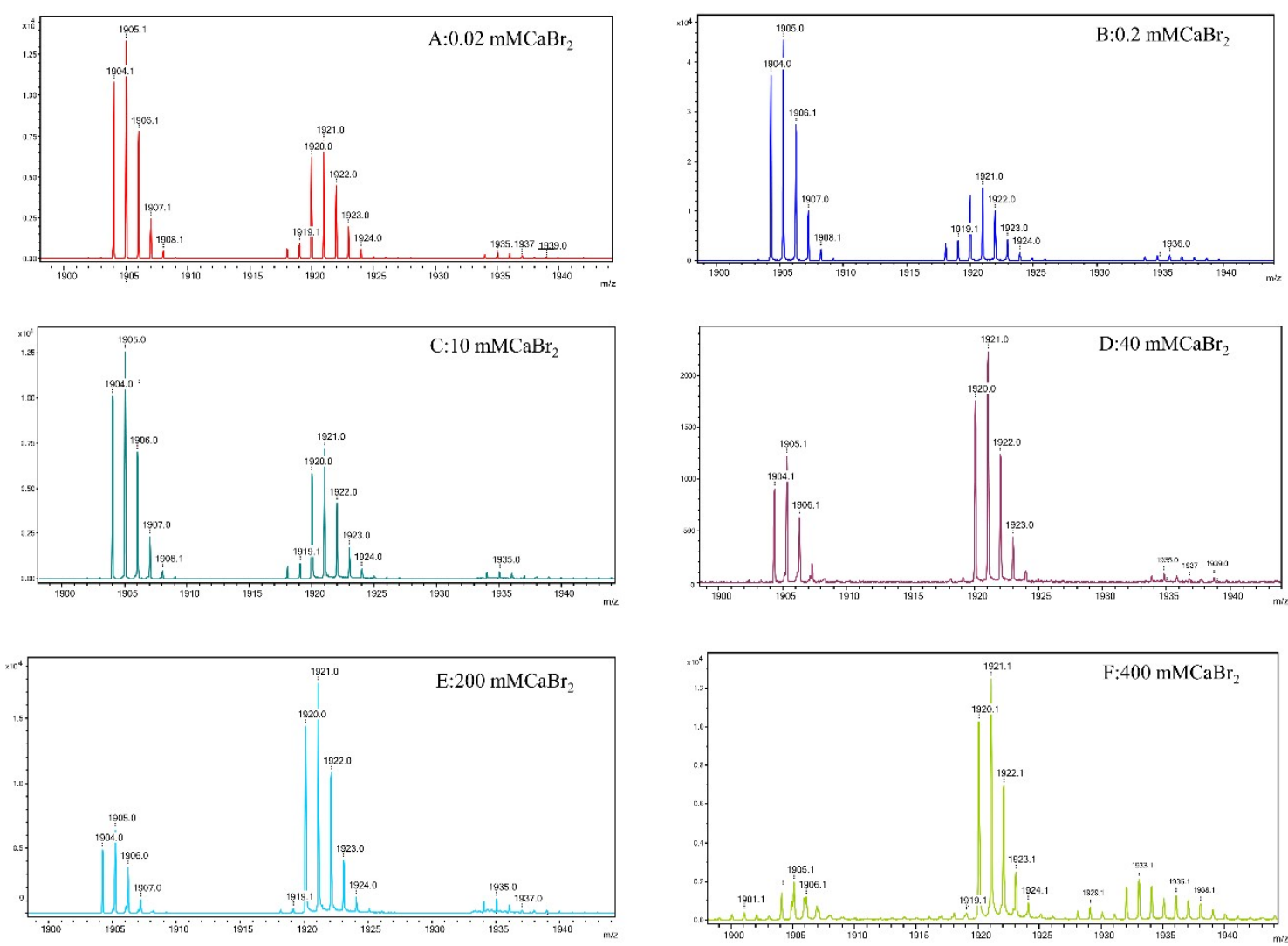

Figure 6. Mass spectra of gramicidin $\mathrm{A}$ in the presence of $\mathrm{CaBr}_{2}$. The samples containing $100 \mu \mathrm{M}$ gramicidin A with $\mathrm{CaBr}_{2}$ at $0.02(\mathrm{~A}), 0.2(\mathbf{B}), 10(\mathbf{C}), 40$ (D), 100 (E) and $400 \mathrm{mM}(\mathbf{F})$ in methanol were mixed with the matrix for mass analyses.

The peaks at $m / z$ 1902-1905 were assigned as (gA dimer/Ca) ${ }^{2+}$, while the peaks at $m / z$ 1920-1921 were assigned as $(\mathrm{gA} \text { monomer } / \mathrm{Ca} / \mathrm{H})^{+}$. In the case of $\mathrm{gA} / \mathrm{CaCl}_{2}$, a new peak at $\mathrm{m} / \mathrm{z} 1936$ was assigned as $(\mathrm{gA} \text { dimer } / \mathrm{Ca}+2 \mathrm{Cl})^{2+}$ or $\left(\mathrm{gA} \text { dimer } / \mathrm{Ca}+4 \mathrm{H}_{2} \mathrm{O}\right)^{2+}$, while this peak was not observed in the cases of $\mathrm{CaBr}_{2}$ (Figure 6) and $\mathrm{CaI}_{2}$ (Figure S2). Due to the high oxidative strength of iodine, we were unable to obtain a reasonable mass for gramicidin $\mathrm{A} / \mathrm{CaI}_{2}$ at concentrations higher than $100 \mathrm{mM}$ (Figure S2).

The molar ratios of the dimer/monomer of gramicidin $\mathrm{A}$ in the presence of different salt concentrations and halides were calculated using the intensity of $1905\left(+1936\right.$ for $\left.\mathrm{CaCl}_{2}\right) / 1920$. The molar ratios of the dimer/monomer are summarized in Table 1 . For $\mathrm{CaCl}_{2}$ and $\mathrm{CaBr}_{2}$, the majority of the gramicidin $\mathrm{A}$ at a low salt concentration, i.e., $0.2 \mathrm{mM}$, was in the dimeric state. The molar ratios of the dimer/monomer were 6.0 and 2.0 for $\mathrm{CaCl}_{2}$ and $\mathrm{CaBr}_{2}$, respectively. At medium salt concentrations, i.e., 10 and $40 \mathrm{mM}$, the molar ratios of the dimer/monomer for $\mathrm{gA}$ in the presence of $\mathrm{CaCl}_{2}$ were two- to four-fold higher than those for $\mathrm{gA}$ in the presence of $\mathrm{CaBr}_{2}$. At a higher salt concentration, i.e., $100 \mathrm{mM}$, gramicidin $\mathrm{A}$ was in the monomeric state for both $\mathrm{CaCl}_{2}$ and $\mathrm{CaBr}_{2}$. In general, $\mathrm{CaBr}_{2}$ induced the formation of the monomer more efficiently than $\mathrm{CaCl}_{2}$ did. This effect was even more profound for $\mathrm{CaI}_{2}$, as most gramicidin A species were monomeric, even at very low $\mathrm{CaI}_{2}$ concentrations. The value of the dimer/monomer ratio might be underestimated. As a $\alpha$-cyano-4-OH cinnamic acid (CHCA) matrix dissolved in $50 \%$ acetonitrile $/ 50 \%$ distilled $\mathrm{H}_{2} \mathrm{O}$ was mixed with the sample in methanol, the acetonitrile/water solvent system favored monomer formation. This may shift the equilibrium toward the monomer and underestimate the dimer/monomer ratio. However, this factor does not affect the conclusion. 
Table 1. Molar ratio of dimer/monomer of gramicidin $\mathrm{A}$ in the presence of $\mathrm{CaCl}_{2}, \mathrm{CaBr}_{2}$ and $\mathrm{CaI}_{2}$ at various concentrations.

\begin{tabular}{cccc}
\hline Salt Concentration & Ratio $\left(\mathbf{C a C l}_{\mathbf{2}}\right)$ & Ratio $\left(\mathbf{C a B r}_{\mathbf{2}}\right)$ & Ratio $\left(\mathbf{C a I}_{\mathbf{2}}\right)$ \\
\hline $0.2 \mathrm{mM}$ & 6.0 & 2.0 & 0.5 \\
$10 \mathrm{mM}$ & 4.2 & 1.5 & 0.25 \\
$40 \mathrm{mM}$ & 2.0 & 0.5 & 0.22 \\
$100 \mathrm{mM}$ & 0.2 & 0.15 & $\mathrm{NA}$ \\
$400 \mathrm{mM}$ & $<0.1$ & $<0.1$ & NA \\
\hline
\end{tabular}

\subsection{Tryptophan Fluorescence Spectroscopy}

Gramicidin A contains four tryptophan residues at its C-terminus. The Trp residues are a good indicator for examining the influence of the microenvironment on gramicidin A's molecular state. The steady-state tryptophan fluorescence spectra of gramicidin A titrated with $\mathrm{CaCl}_{2}$ and $\mathrm{CaBr}_{2}$ are shown in Figure 7A,B, respectively. For both $\mathrm{CaCl}_{2}$ and $\mathrm{CaBr}_{2}$, there is a fluorescent peak located at around $350 \mathrm{~nm}$. At calcium salt concentrations $\leq 10 \mathrm{mM}$, the fluorescence intensity increased with an increase in the calcium salt concentration, whereas at calcium salt concentration $>10 \mathrm{mM}$, the intensity gradually decreased with an increase in the salt concentration. The peak wavelength also underwent a redshift with an increase in salt concentration at the salt concentrations $\leq 10 \mathrm{mM}$ and a blueshift with an increase in salt concentration at the salt concentrations $>10 \mathrm{mM}$, indicating that calcium halide salts may have an impact not only on the gramicidin A backbone but also on the indole rings of the four Trp residues. The blueshift of the wavelength and decrease in intensity at high concentrations of Ca salts, particularly $\mathrm{CaBr}_{2}$ and $\mathrm{CaI}_{2}$, may suggest that gramicidin $\mathrm{A}$ undergoes denaturation [31,32]. This reinforces the results obtained in the NMR studies.
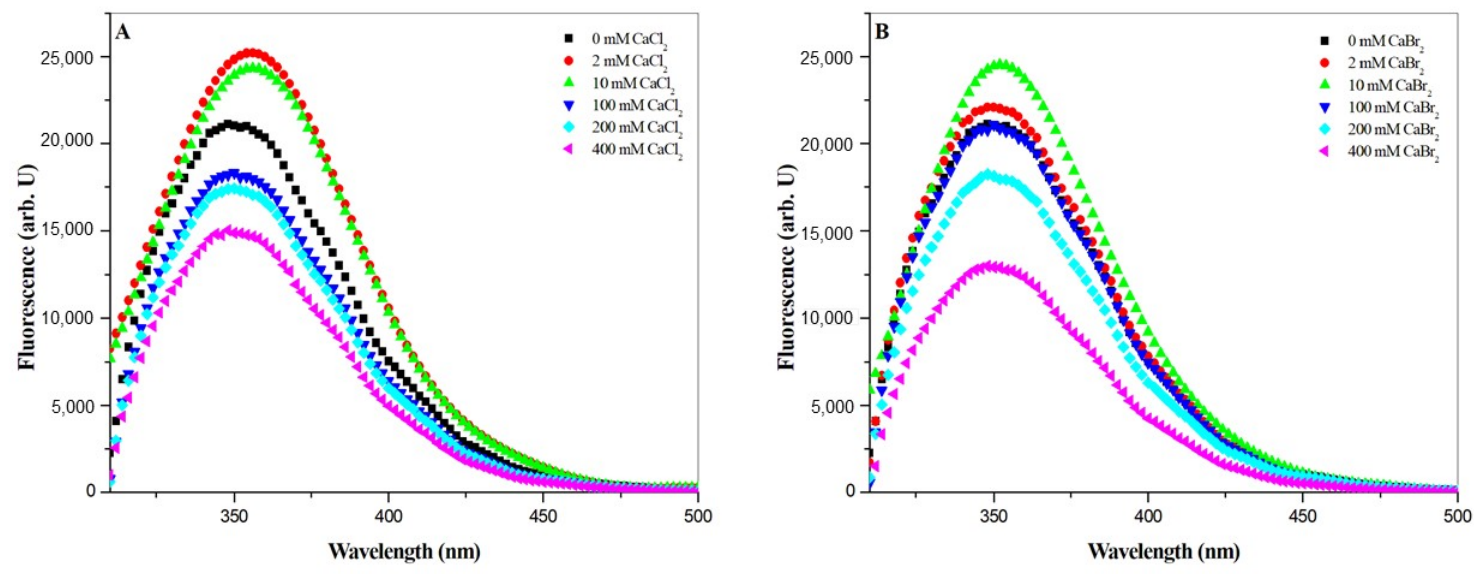

Figure 7. Steady-state fluorescence spectra of gramicidin $\mathrm{A}$ in the presence of $(\mathbf{A}) \mathrm{CaCl}_{2}$ and (B) $\mathrm{CaBr}_{2}$. One hundred $\mu \mathrm{M}$ of gramicidin $\mathrm{A}$ titrated with $\mathrm{CaCl}_{2}$ and $\mathrm{CaBr}_{2}$ in methanol. In the fluorescence spectra, the concentration of $\mathrm{CaCl}_{2}$ or $\mathrm{CaBr} 2$ increases from top to bottom.

\subsection{Antimicrobial Activities}

Our spectroscopic studies demonstrate that the backbone conformation and side-chain microenvironment of gramicidin A were profoundly affected by the concentration of calcium and the types of halide anion. We then examined the antimicrobial activity of gramicidin $\mathrm{A}$ in the presence of $\mathrm{CaCl}_{2}$. Figure 8 shows the antimicrobial activity of gramicidin A with different concentrations of $\mathrm{CaCl}_{2}$ against Staphylococcus aureus at different bacterial growth phases. The antimicrobial activity of gramicidin A was inhibited in the pretreatment with $400 \mathrm{mM} \mathrm{CaCl}_{2}$ compared to the control (without $\mathrm{gA}$ ) at all growth phases. In the lag phase, the growth of $S$. aureus was significantly inhibited by gramicidin $\mathrm{A}$ with or without $\mathrm{CaCl}_{2}$. In the exponential phase, the antimicrobial activity of gramicidin 
A was significantly inhibited in the pretreatment with $100 \mathrm{mM} \mathrm{CaCl} 2$ or in the absence of $\mathrm{CaCl}_{2}$ compared to that with the pretreatment of 0.2 and $10 \mathrm{mM} \mathrm{CaCl}_{2}$. In the late-exponential-to-stationary phase, the antimicrobial activity of gramicidin $\mathrm{A}$ in the pretreatment with $100 \mathrm{mM} \mathrm{CaCl} 2$ was more effective than that in the pretreatments with other $\mathrm{CaCl}_{2}$ concentrations. The antimicrobial activity of gramicidin $\mathrm{A}$ was similar in $\mathrm{CaBr}_{2}$. The most effective concentration regarding antimicrobial activity for $\mathrm{CaBr}_{2}$ was $10 \mathrm{mM}$ rather than $100 \mathrm{mM}$ (Figure S3).
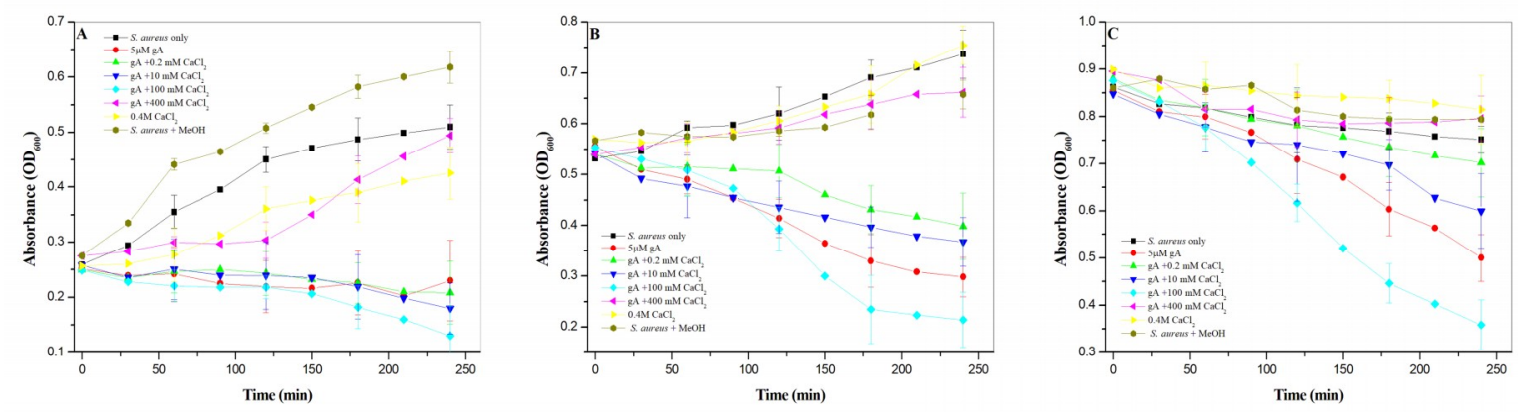

Figure 8. The antimicrobial activity of gramicidin $\mathrm{A}$ in the presence of $\mathrm{CaCl}_{2}(\mathbf{A}-\mathbf{C})$. In the antimicrobial activity assay, stock solutions were prepared containing $100 \mu \mathrm{M}$ gramicidin A with $0,0.2,10,100$ and $400 \mathrm{mM} \mathrm{CaCl}_{2}$ in methanol. The stock solutions were then diluted into $S$. aureus culture with a final solution containing $5 \mu \mathrm{M}$ gramicidin A. (A), lag phase; (B), exponential phase; and (C), stationary phase.

\section{Discussion}

The effect of calcium cations on gramicidin A's conformation was previously studied using infrared (IR) and CD spectroscopic techniques [18-20,33]. However, the detailed molecular and conformational states of gramicidin A under the effects of calcium cations and halide anions required further verification. In the present study, we applied state-of-the-art mass, NMR and fluorescence spectroscopy to clarify the detailed molecular and structural states of gramicidin A in the presence of calcium cations and halide anions. We used the obtained structural and molecular information to examine the effect of calcium halides on the antimicrobial activity of gramicidin A.

Our results obtained from CD and NMR spectroscopies are similar to those from the studies in $\mathrm{CaCl}_{2}$ by Wallace and colleagues $[18,19]$. According to the combination of CD and NMR spectra, the effect of calcium halides on the conformation of gramicidin A reached a turning point at the concentration of $10 \mathrm{mM}$ for $\mathrm{CaCl}_{2}, \mathrm{CaBr}_{2}$ and $\mathrm{CaI}_{2}$, indicating that gramicidin $\mathrm{A}$ may exist in two molecular states, State 1 at salt concentrations $\leq 10 \mathrm{mM}$ and State 2 at salt concentration $>10 \mathrm{mM}$. The observations obtained with CD and NMR are consistent with the fluorescence spectroscopic studies. In the fluorescence spectra, the intensity increased with an increase in the salt concentration, demonstrated a redshift in wavelength at concentrations $\leq 10 \mathrm{mM}$, and decreased with an increase in concentration with a blueshift in wavelength at concentrations $>10 \mathrm{mM}$. These results reinforce that there are two states for gramicidin A upon the titration of calcium halides.

From the CD and NMR spectra, we demonstrated that the main effect of calcium salt is to drive a mixture of conformers into a single species in State 1. This is consistent with the previous study by Chen and Wallace [19]. In addition, we further found that this effect on gramicidin A conformation was dependent on the calcium concentration and independent of the types of halide anions. The progress of the NMR spectral change vs. concentration was similar for all different halide anions, indicating that the conformational change of gramicidin $\mathrm{A}$ is only induced by $\mathrm{Ca}^{2+}$ cations. The 1D NMR spectra of gramicidin $\mathrm{A}$ at $10 \mathrm{mM}$ showed a similar resonance pattern at the amide proton region for $\mathrm{CaCl}_{2}$, $\mathrm{CaBr}_{2}$ and $\mathrm{CaI}_{2}$. These NMR spectra are similar to those in a previous NMR study [20], suggesting that the conformation of gramicidin A may form a left-handed parallel double helix under this condition.

The most interesting findings in the present study are from the mass spectroscopy analyses. The analyses of the mass spectra for all calcium halides suggest that the molecular state of gramicidin 
A is a mixture of the dimer and monomer in State 1. The major form of gramicidin A exists in a dimeric form for both $\mathrm{CaCl}_{2}$ and $\mathrm{CaBr}_{2}$ but not for $\mathrm{CaI}_{2}$. The dimer content decreased with an increase in the salt concentration, indicating that the addition of the calcium salt drove a dissociation of gramicidin A dimers into monomers. The dissociation of the gramicidin A dimer was more effective with $\mathrm{CaI}_{2}$ or $\mathrm{CaBr}_{2}$ than with $\mathrm{CaCl}_{2}$, suggesting that the dissociation is highly dependent on the type of halide anion. The order by dissociation ability is $\mathrm{I}^{-}>\mathrm{Br}^{-}>\mathrm{Cl}^{-}$.

The reason for this phenomenon is not apparent. A possible reason may be the ionic radii of the halide anion. A peak at $m / z 1936$ assigned as $\left(\mathrm{gA} \text { dimer } / \mathrm{Ca}^{+}+2 \mathrm{Cl}\right)^{2+}$ only appeared in the mass spectrum of $\mathrm{CaCl}_{2}$ but not in the cases of $\mathrm{CaBr}_{2}$ and $\mathrm{CaI}_{2}$. A similar observation was reported by Zhou and colleagues [34]. These results suggest that the gramicidin A dimer may interact only with $\mathrm{Cl}^{-}$, possibly inside the pore, but not with $\mathrm{Br}^{-}$or $\mathrm{I}^{-}$. As the ionic radii of $\mathrm{Br}^{-}$and $\mathrm{I}^{-}$are larger than that of $\mathrm{Cl}^{-}$, both the $\mathrm{Br}^{-}$and $\mathrm{I}^{-}$anions are too large to accommodate them inside the dimer pore of gramicidin $\mathrm{A}$. The interaction of $\mathrm{Cl}^{-}$ions with gramicidin A may stabilize the dimer and reduce the dimer-to-monomer conversion rate. Therefore, the molar ratio of dimer/monomer in $\mathrm{CaCl}_{2}$ is much higher than the molar ratios in $\mathrm{CaBr}_{2}$ and $\mathrm{CaI}_{2}$. However, the exact reason needs to be further verified with a detailed structure.

In State 2, our CD spectra are also similar to those in a study by Chen and Wallace [19]. The two negative $\mathrm{CD}$ peaks gradually converted into a single positive peak in $\mathrm{CaCl}_{2}$, indicating that gramicidin A may adopt a different conformation. Chen and Wallace proposed that gramicidin A may be a mixture of unstructured and structured monomers in this condition. Analyses of the mass spectra indicate that the molar ratios of dimer/monomer were all less than 1 for most calcium halides except for $40 \mathrm{mM}$ $\mathrm{CaCl}_{2}$. Our results support the augment that the molecular state of gramicidin A should be a monomer in State 2. Similarly, the formation of monomers is also anion-type dependent.

We examined the structural state of gramicidin A using NMR spectroscopy. The results showed that the effect of halide anions on the gramicidin A's conformation was very different. Gramicidin $\mathrm{A}$ is a mixture of unstructured and structured monomers in the presence of $200 \mathrm{mM} \mathrm{CaCl}_{2}$. This is consistent with a previous study by Chen and Wallace [19]. The conformation of gramicidin A in $200 \mathrm{mM} \mathrm{CaBr}_{2}$ was more unstructured compared to that in $200 \mathrm{mM} \mathrm{CaCl}_{2}$. The unstructured effect was even more profound with the addition of $200 \mathrm{mM} \mathrm{CaI}_{2}$, indicating that $\mathrm{Br}^{-}$and $\mathrm{I}^{-}$anions may induce denaturation in gramicidin $\mathrm{A}$. The denaturing effect of halide anions on gramicidin $\mathrm{A}$ is well correlated with the trend of the dimer-to-monomer dissociation of gramicidin $\mathrm{A}: \mathrm{I}^{-}>\mathrm{Br}^{-}>\mathrm{Cl}^{-}$.

The denaturation of gramicidin A induced by halide was further confirmed by the fluorescence spectroscopy. During protein unfolding, the fluorescence spectrum usually undergoes a blueshift in the maximum wavelength and a decrease in the intensity [31,32,35]. In State 2, the fluorescence intensity decreased with an increase in the salt concentration, and the maximum wavelength underwent a blueshift with an increase in the salt concentration., suggesting that the gramicidin A monomer may be denatured by halide anions. Taken together, these results suggest that the halide anions, particularly $\mathrm{Br}^{-}$and $\mathrm{I}^{-}$, may play a role in the denaturation of gramicidin A. The denaturation ability of halide anions, particularly $\mathrm{Br}^{-}$and $\mathrm{I}^{-}$, could be taken to account for the nature of the dissociation of gramicidin A.

The antimicrobial activity of gramicidin A increased with an increase in the calcium concentration but not for $400 \mathrm{mM} \mathrm{CaCl}_{2}$. At $400 \mathrm{mMCaCl}_{2}$, the antimicrobial activity of gramicidin A was significantly inhibited. The antimicrobial activity of gramicidin $\mathrm{A}$ is in the order $100 \mathrm{mM} \mathrm{CaCl}{ }_{2}>0 \mathrm{mM}$ $\mathrm{CaCl}_{2}>10 \mathrm{mMCaCl}_{2} \approx 0.2 \mathrm{mM} \mathrm{CaCl}_{2}>>400 \mathrm{mMCaCl}_{2}$. The molecular states of gramicidin $\mathrm{A} / \mathrm{CaCl}_{2}$ complexes in methanol may be changed when they are added to culture medium, as the addition of the gramicidin A in methanol into culture medium may drive the conversion of dimers into monomers. Therefore, the antimicrobial activity may have been overestimated in the present study. However, this does not affect the present conclusion.

The results obtained for the antimicrobial activity vs. $\mathrm{CaCl}_{2}$ concentration suggest that the monomeric gramicidin A had the most effective inhibitory ability against bacterial growth. A previous 
study by Jadhay et al. suggested that gramicidin A in channel form mediated the most effective antimicrobial activity against Gram-positive bacteria [29]. The formation of gramicidin channels in membranes is highly dependent on the environment [36,37]. In $100 \mathrm{mM} \mathrm{CaCl}_{2}$, the main form of gramicidin A is monomeric. Gramicidin A in the presence of $100 \mathrm{mM} \mathrm{CaCl}_{2}$ can readily form ion channels in bacterial cell walls and, hence, shows the most effective antimicrobial activity. On the other hand, the major form of gramicidin $\mathrm{A}$ is dimeric in $0,0.2$ and $10 \mathrm{mM} \mathrm{CaCl}_{2}$. When bacteria are treated with gramicidin $\mathrm{A}$ in these conditions, it takes time to convert the dimers into monomers. Therefore, gramicidin $\mathrm{A}$ in these conditions was less effective in terms of antimicrobial activity when compared to the case at $100 \mathrm{mM} \mathrm{CaCl}_{2}$.

The case of gramicidin A in $400 \mathrm{mM} \mathrm{CaCl}_{2}$ was different from that at $100 \mathrm{mM} \mathrm{CaCl}_{2}$. Gramicidin A may exist largely in the denatured state in $400 \mathrm{mM} \mathrm{CaCl}_{2}$. This may cause the loss of the antimicrobial function. However, we could not exclude the possibility that the decrease in antimicrobial activity may have been due to the blocking of the gramicidin A channel by the high concentration of calcium cations, as calcium cations have been shown to interact with gramicidin A channels at the mouth sites and block the conduction of monovalent cations across the cell membrane [22,33,38].

In conclusion, we demonstrate that the molecular state of gramicidin A is highly influenced by the concentration of calcium salt and the types of halide anion. Gramicidin A exists in two molecular states. In State 1 (concentrations $\leq 10 \mathrm{mM}$ ), the majority of gramicidin A forms a dimer. The addition of calcium salt converts the four conformers into a single species that forms a left-handed parallel double helical structure. In State 2, the addition of calcium salt induces a dissociation of dimers into monomers and a further denaturation of the structured monomers into the unstructured monomers. The abilities of dissociation and denaturation are highly dependent on the type of halide anion. Regarding antimicrobial activity, gramicidin A in the structured monomeric state showed the most effective antimicrobial activity. As the molecular state of gramicidin A is highly dependent on the dissociation ability of the halide anions, the dissociation rate can determine the molecular state and antimicrobial activity. Taken together, our study suggests that the molecular state may play a vital role in the antimicrobial activity.

\section{Materials and Methods}

\subsection{Materials}

Gramicidin A was purchased from Merck (Darmstadt, Germany). Spectrograde methanol and methyl-d3-alcohol were purchased from Sigma-Aldrich (St. Louis, MO, USA). Calcium chloride, calcium bromide and calcium iodide were purchased from Sigma-Aldrich (St. Louis, MO, USA). All chemicals were reagent grade and used without further purification.

\subsection{Circular Dichroism (CD) Spectroscopy}

Circular dichroism spectra were recorded using a J-815 CD spectrometer (JASCO International Co. Ltd., Tokyo, Japan) or a Chirascan-plus qCD spectrometer (Applied Photophysics, Surrey, UK). All measurements were performed in a quartz cell with a pathlength of $0.1 \mathrm{~mm}$. The CD spectra of $100 \mu \mathrm{M}$ gramicidin $\mathrm{A}$ in the presence of calcium salts with a designated concentration were collected from 200 to $250 \mathrm{~nm}$ with a $0.5 \mathrm{~nm}$ interval at $25^{\circ} \mathrm{C}$. The reported circular dichroism spectra were corrected with baseline using methanol containing the same concentration of salt and smoothed using a Savitsky-Golay function in Origin 6.0.

\subsection{Mass Spectroscopy}

Gramicidin A samples (100 $\mu \mathrm{M}$ each) were mixed with $\mathrm{CaCl}_{2}, \mathrm{CaBr}_{2}$ or $\mathrm{CaI}_{2}$ at ten different concentrations $(0,0.02,0.07,0.2,2,10,40,100,200$ and $300 \mathrm{mM})$ dissolved in either $\mathrm{D}_{2} \mathrm{O}$ or methanol. These samples were analyzed using MALDI-TOF mass spectrometry. 
The matrix used for the analysis was $\alpha$-cyano-4-OH cinnamic acid (CHCA). A $10 \mathrm{mg}$ amount of CHCA was weighed and dissolved in $1.0 \mathrm{~mL}$ of $50 \%$ acetonitrile, $50 \%$ distilled $\mathrm{H}_{2} \mathrm{O}$ and $0.1 \%$ trifluoroacetic acid (TFA). The gramicidin A samples were then mixed with the CHCA matrix by adding $1 \mu \mathrm{L}$ of the sample with $1 \mu \mathrm{L}$ of the CHCA matrix. After mixing the sample and matrix well, $1 \mu \mathrm{L}$ of the resulting mixtures were then spotted on to the MALDI plate for analysis. The MALDI spectra in the reflection mode were recorded with a Bruker New ultrafleXtreme ${ }^{\mathrm{TM}}$ MALDI-TOF/TOF mass spectrometer from Bruker Daltonik, Bremen, Germany, at the Academia Sinica Institute of Chemistry Mass Spectrometry Center.

\subsection{Nuclear Magnetic Resonance (NMR) Spectroscopy}

We analyzed gramicidin $\mathrm{A}(100 \mu \mathrm{M})$ with $\mathrm{CaCl}_{2}, \mathrm{CaBr}_{2}$ or $\mathrm{CaI}_{2}(0.02,0.2,10$ and $200 \mathrm{mM})$ dissolved in $90 \% \mathrm{~d} 4$-methanol $/ 10 \%$ methanol using solution NMR. The NMR spectra were obtained using a Bruker DRX-500 NMR spectrometer equipped with a TXI z-gradient $(1 \mathrm{H}, 13 \mathrm{C}, 15 \mathrm{~N})$ probe at $300 \mathrm{~K}$ (Bruker Biospin $\mathrm{GmbH}$, Rheinstetten, Germany). In these spectra, the $\mathrm{OH}$ signal of methanol was suppressed using the presaturation method. All the NMR spectra were phased and baseline-corrected using the Topspin software (version 3.2.2; Bruker Biospin GmbH, Rheinstetten, Germany) and referenced to the chemical shift of methanol at $3.3 \mathrm{ppm}$.

\subsection{Steady-State Fluorescence Measurements}

All steady-state fluorescence measurements were performed with a Jasco FP-6500 fluorescence spectrometer (Tokyo, Japan) equipped with a water circulator and stirring accessory. The emission spectra of $100 \mu \mathrm{M}$ gramicidin A in the presence of calcium halide salts at designed concentrations were recorded from 310 to $500 \mathrm{~nm}$ with an excitation wavelength of $290 \mathrm{~nm}$. The bandwidths for excitation and emission were 3 and $5 \mathrm{~nm}$, respectively. All measurements were performed in a quartz cell with a path length of $1 \mathrm{~cm}$. The background spectra of the salts alone were taken first for later subtraction. The reported spectra were the averages obtained from at least three individual samples and three repeated measurements of each sample. All measurements were carried out at $25.0 \pm 0.5^{\circ} \mathrm{C}$.

\subsection{Bacterial Growth Assay}

Staphylococcus aureus (S. aureus) (ATCC-25923) was kindly provided by Dr. J.W. Liou in Tzu Chi University. The S. aureus was grown in Luria-Bertani (LB) broth medium in a $250 \mathrm{~mL}$ flask at $37^{\circ} \mathrm{C}$ overnight. This overnight $S$. aureus culture was then diluted to $\mathrm{OD}_{600}=0.1$ with LB medium. This $S$. aureus was then grown to lag phase $\left(\mathrm{OD}_{600}=0.2\right)$, exponential phase $\left(\mathrm{OD}_{600}=0.6\right)$ and late-exponential-to-stationary phase $\left(\mathrm{OD}_{600}=1.5\right)$. At the designated growth phase, $5 \mathrm{~mL}$ of $S$. aureus culture was treated at $5 \%(V / V)$ with a stock solution containing gramicidin A (a final concentration of $5 \mu \mathrm{M}$ ) and $\mathrm{CaCl}_{2}$. The stock solution was prepared in the same way as used for the spectroscopic studies, in which a final concentration of $100 \mu \mathrm{M}$ gramicidin A was dissolved in methanol containing $0,0.2,10,100$ and $400 \mathrm{mM}$ of $\mathrm{CaCl}_{2}$. The $S$. aureus samples treated with or without gramicidin $\mathrm{A} / \mathrm{CaCl}_{2}$ were incubated at $37^{\circ} \mathrm{C}$. The optical density at a wavelength of $600 \mathrm{~nm},\left(\mathrm{OD}_{600}\right)$, was used to determine the growth curve of S. aureus using a microplate reader (FlexStation 3, MD) every $30 \mathrm{~min}$.

Supplementary Materials: Supplementary materials can be found at http://www.mdpi.com/1422-0067/21/17/ 6177/s1.

Author Contributions: Conceptualization, Y.-C.C.; data curation, K.D.C., C.-J.L., D.-L.M.T., S.-T.F., S.-H.H. and Y.-C.C.; formal analysis, K.D.C., C.-J.L. and Y.-C.C.; investigation, K.D.C., C.-J.L., D.-L.M.T., S.-T.F. and Y.-C.C.; methodology, D.-L.M.T., Y.-H.L. and Y.-C.C.; project administration, Y.-C.C.; software, C.-J.L., D.-L.M.T., Y.-H.L. and Y.-C.C.; validation, Y.-C.C.; visualization, Y.-C.C.; writing-original draft, Y.-C.C.; writing-review and editing, Y.-C.C. All authors have read and agreed to the published version of the manuscript.

Funding: This work was supported by grants from the Ministry of Science and Technology, Taiwan (MOST108-2113-M-715-001 and MOST107-2113-M715-001 to Y.-C.C.), and Mackay Medical College (1041B05 and 1071B05 to Y.-C.C.). 
Acknowledgments: We would also like to thank the National Synchrotron Radiation Center and Biophysics Core Facility, Academia Sinica, for providing the CD spectrometer.

Conflicts of Interest: The authors declare no competing financial interests.

\section{References}

1. Hotchkiss, R.D.; Dubos, R.J. Fractionation of bactericidal agent from cultures of a soil bacillus. J. Biol. Chem. 1940, 132, 791-792.

2. Sarges, R.; Witkop, B.; Gramicidin, A.V. The structure of valine and isoleucine-gramicidin A. J. Am. Chem. Soc. 1965, 87, 2011-2020. [CrossRef]

3. Weinstein, S.; Wallace, B.A.; Morrow, J.S.; Veatch, W.R. Conformation of the gramicidin a transmembrane channel: A 13C nuclear magnetic resonance study of 13C-enriched gramicidin in phosphatidylcholine vesicles. J. Mol. Biol. 1980, 143, 1-19. [CrossRef]

4. Wallace, B.A. Gramicidin channels and pores. Annu. Rev. Biophys. Biophys. Chem. 1990, 19, 127-157. [CrossRef]

5. Sychev, S.V.; Barsukov, L.I.; Ivanov, V.T. The double pi-pi-5.6 helix of gramicidin A predominates in unsaturated lipid-membranes. Eur. Biophys. J. 1993, 22, 279-298. [CrossRef]

6. Wallace, B.A. Gramicidin adopts distinctly different conformations in organic solvents and in membranes. Biopolymers 1983, 22, 397-402. [CrossRef]

7. Pascal, S.M.; Cross, T.A. Structure of an isolated gramicidin a double helical species by high resolution nuclear magnetic resonance. J. Mol. Biol. 1992, 226, 1101-1109. [CrossRef]

8. Chen, Y.; Wallace, B.A. Solvent effects on the conformation and far UV CD spectra of gramicidin. Biopolymers 1997, 42, 771-781. [CrossRef]

9. Veatch, W.R.; Blout, E.R. The aggregation of gramicidin A in solution. Biochemistry 1974, 13, 5257-5264. [CrossRef]

10. Bouchard, M.; Benjamin, D.R.; Tito, P.; Robinson, C.V.; Dobson, C.M. Solvent effects on the conformation of the transmembrane peptide gramicidin A: Insights from electrospray ionization mass spectrometry. Biophys. J. 2007, 78, 1010-1017. [CrossRef]

11. Urry, D.W.; Mayers, D.F.; Haider, J. Spectroscopic studies on the conformation of gramicidin A. Evidence for a new helical conformation. Biochemistry 1972,11,487-493. [CrossRef]

12. Wallace, B.A. Recent Advances in the High Resolution Structures of Bacterial Channels: Gramicidin A. J. Struct. Biol. 1998, 121, 123-141. [CrossRef]

13. Hinton, J.F.; Koeppe, R.E.; Shungu, D.; Whaley, W.L.; Paczkowski, J.A.; Millett, F.S. Equilibrium binding constants for the group I metal cations with gramicidin-A determined by competition studies and Tl+-205 nuclear magnetic resonance spectroscopy. Biophys. J. 1986, 49, 571-577. [CrossRef]

14. Pullman, A. Contribution of theoretical chemistry to the study of ion transport through membranes. Chem. Rev. 1991, 91, 793-812. [CrossRef]

15. Wallace, B.A.; Ravikumar, K. The gramicidin pore: Crystal structure of a cesium complex. Science 1988, 241, 182-187. [CrossRef]

16. Doyle, D.A.; Wallace, B.A. Caesium-binding sites in the gramicidin pore. Biochem. Soc. Trans. 1994, 22, 1043-1045. [CrossRef]

17. Chen, Y.; Wallace, B.A. Binding of alkaline cations to the double-helical form of gramicidin. Biophys. J. 1996, 71, 163-170. [CrossRef]

18. Doyle, D.A.; Wallace, B.A. Shifting the Equilibrium Mixture of Gramicidin Double Helices toward a Single Conformation with Multivalent Cationic Salts. Biophys. J. 1996, 75, 635-640. [CrossRef]

19. Chen, Y.; Wallace, B.A. The effects of calcium ions on double helical forms of gramicidin. Eur. Biophys. J. 1997, 26, 299-306. [CrossRef]

20. Chen, Y.; Tucker, A.; Wallace, B.A. Solution structure of a parallel left-handed double-helical gramicidin A determined by 2D 1H-NMR. J. Mol. Biol. 1996, 264, 757-769. [CrossRef]

21. Wallace, B.A.; Veatch, W.R.; Blout, E.R. Conformation of gramicidin A in phospholipid vesicles: Circular dichroism studies of effects of ion-binding, chemical modification, and lipid structure. Biochemistry. 1981, 20, 5754-5760. [CrossRef]

22. Carvalho, G.A.; Olivares-Ortega, C.; Soto-Arriaza, M.A.; Carmona-Ribeiro, A.M. Interaction of gramicidin with DPPC/DODAB bilayer fragments. Biochim. Biophys. Acta 2012, 1818, 3064-3071. [CrossRef] 
23. Lin, T.H.; Huang, H.B.; Wei, H.A.; Shiao, S.H.; Chen, Y.C. The effect of temperature and lipid on the conformational transition of gramicidin A in lipid vesicles. Biopolymers 2005, 78, 179-186. [CrossRef]

24. Sychev, S.V.; Ivanov, V.T. Large scale conformational transitions in $\beta$-structural motif of gramicidin A: Kinetic analysis based on CD and FT-IR data. J. Pept. Sci. 2014, 20, 657-667. [CrossRef]

25. Stevenson, P.; Tokmakoff, A. Time-resolved measurements of an ion channel conformational change driven by a membrane phase transition. Proc. Natl. Acad. Sci. USA 2017, 114, 10840-10845. [CrossRef]

26. Zerfas, B.L.; Joo, Y.; Gao, J. Gramicidin A Mutants with Antibiotic Activity against both Gram-Positive and Gram-Negative Bacteria. Chem. Med. Chem. 2016, 11, 629-636. [CrossRef]

27. Huang, H.W. Peptide-lipid interactions and mechanisms of antimicrobial peptides. Novartis Found Symp. 1999, 225, 188-206.

28. Liou, J.W.; Hung, Y.J.; Chen, Y.C. The antimicrobial activity of gramicidin A is associated with hydroxyl radical formation. PLoS ONE 2015, 10, e0117065. [CrossRef]

29. Jadhav, K.B.; Stein, C.; Makarewicz, O.; Pradel, G.; Lichtenecker, R.J.; Sack, H.; Heinemann, S.H.; Arndt, H.D. Bioactivity of topologically confined gramicidin A dimers. Bioorg. Med. Chem. 2017, 25, 261-268. [CrossRef]

30. Arseniev, A.S.; Barsukov, I.L.; Bystrov, V.F. NMR solution structure of gramicidin a complex with caesium cations. FEBS Lett. 1985, 180, 33-39. [CrossRef]

31. Eftink, E.A. The use of fluorescence methods to monitor unfolding transitions in proteins. Biophys. J. 1994, 66, 482-501. [CrossRef]

32. Monsellier, E.; Bedouelle, H. Quantitative measurement of protein stability from unfolding equilibria monitored with fluorescence maximum wavelength. Protein Eng. Des. Sel. 2005, 18, 445-456. [CrossRef]

33. Heitz, F.; Gavach, C. Ca++-gramicidin A interactions and blocking effects on the ionic channel. Biophys. Chem. 1983, 18, 153-163. [CrossRef]

34. Sudhir, P.R.; Wu, H.F.; Zhou, Z.C. An application of electrospray ionization tandem mass spectrometry to probe the interaction of $\mathrm{Ca}^{2+} / \mathrm{Mg}^{2+} / \mathrm{Zn}^{2+}$ and $\mathrm{Cl}^{-}$with gramicidin A. Rapid Commun. Mass Spectrom. 2005, 19, 1517-1521. [CrossRef]

35. Ghisaidoobe, A.B.T.; Chung, S.J. Intrinsic Tryptophan Fluorescence in the detection and Analysis of Proteins: A Focus on Forster resonance Energy Transfer Techniques. Int. J. Mol. Sci. 2014, 15, 22518-22538. [CrossRef]

36. Bañó, M.C.; Braco, L.; Abad, C. HPLC study on the 'history' dependence of gramicidin a conformation in phospholipid model membranes. FEBS Lett. 1989, 250, 67-71. [CrossRef]

37. LoGrasso, P.V.; Moll, F.; Cross, T.A. Solvent history dependence of gramicidin a conformations in hydrated lipid bilayers. Biophys. J. 1988, 54, 259-267. [CrossRef]

38. Olah, G.A.; Huang, H.W.; Liu, W.H.; Wu, Y.L. Location of ion binding sites in the gramicidin channel by X-ray diffraction. J. Mol. Biol. 1991, 218, 847-858. [CrossRef] 